\title{
Supplementing material for "A new top boundary condition for modeling surface diffusive exchange of a generic volatile tracer: theoretical analysis and application to soil evaporation"
}

J. Y. Tang and W. J. Riley

Earth Sciences Division, Lawrence Berkeley National Lab (LBL), Berkeley, CA, United States

Correspondence to: jinyuntang@lbl.gov

\section{S1 The capillary diffusivity}

According to Darcy's law, the advective liquid flux of a passive tracer is

$$
Q=-K \frac{\partial h}{\partial z} C_{w}
$$

where $C_{w}$ (mol tracer $\mathrm{m}^{-3}$ water) is the aqueous concentration of the tracer and $K$ $\left(\mathrm{m} \mathrm{s}^{-1}\right)$ is the hydraulic conductivity, and $h(\mathrm{~m})$ is the hydraulic head, which is a sum of the soil matric potential $\psi(\mathrm{m})$ and the gravity potential $z(\mathrm{~m})$. In Eq. (S1.1) and below, we assume that $z$ is positive upward.

For a non-isothermal parameterization, $\psi$ is a function of temperature $T(\mathrm{~K})$ and moisture $\theta\left(\mathrm{m}^{3} \mathrm{~m}^{-3}\right)$. Then Eq. (S1.1) can be re-written as 


$$
Q=-K\left(\frac{\partial \psi}{\partial z}+\frac{\partial \psi}{\partial T} \frac{\partial T}{\partial z}+1\right) C_{w}
$$

or as

$$
Q=-K\left[\frac{\partial \psi}{\partial \theta}+\left(\frac{\partial \psi}{\partial T} \frac{\partial T}{\partial z}+1\right) \frac{\partial z}{\partial \theta}\right] \frac{\partial \theta}{\partial z} C_{w}
$$

When Eq. (S1.3) is applied at the top soil control volume (TSCV), one has

$$
Q=-K\left[\frac{\partial \psi}{\partial \theta}+\left(\frac{\partial \psi}{\partial T} \frac{\partial T}{\partial z}+1\right) \frac{\partial z}{\partial \theta}\right] \frac{\theta_{s}-\theta_{1}}{\Delta z / 2} C_{w, 1}
$$

By invoking the fast equilibrium assumption between aqueous and gaseous phase, one finds

$$
\frac{C_{w, S}}{C_{w, 1}}=\frac{\mathrm{B} C_{g, s}}{\mathrm{~B} C_{g, 1}}=\frac{C_{g, s}}{C_{g, 1}}
$$

where B is the Bunsen solubility coefficient, which is assumed constant across the TSCV. Here and below, the subscript " $g$ " indicates a gaseous tracer; subscript " $s$ " indicates the tracer is defined at the soil-air interface, and subscript "1" indicates the tracer is defined at the center of the TSCV.

Since Eq. (S1.5) is applicable to an arbitrary tracer, its application to the water molecule leads to 


$$
C_{g, s}=\frac{\rho_{a t m} q_{s}}{M_{w}}, C_{g, 1}=\frac{\rho_{a t m} q_{1}}{M_{w}}, C_{w, 1}=\frac{\rho_{l, 1}}{M_{w}}, C_{w, s}=\frac{\rho_{l, s}}{M_{w}}
$$

where $\rho_{\text {atm }}\left(\mathrm{kg} \mathrm{m}^{-3}\right)$ is the atmospheric air density, $q_{x}, x=1, s$ (g water vapor $\mathrm{g}^{-1}$ air) is the specific humidity at either the center of TSCV (when $x=1$ ) or the surface (when $x=s$ ), $M_{w}\left(\mathrm{~kg} \mathrm{~mol}^{-1}\right)$ is the molar mass of water, and $\rho_{l, x}, x=1, s\left(\mathrm{~kg} \mathrm{~m}^{-3}\right)$ is the liquid water density at either the center of TSCV (when $x=1$ ) or the surface (when $x=s$ ).

Define the Bunsen solubility coefficient for the water molecule as

$$
\mathrm{B}=\frac{C_{w, 1}}{C_{g, 1}}=\frac{\rho_{l, 1}}{\rho_{a t m} q_{1}}
$$

Because the Bunsen solubility coefficient is assumed uniform across the topsoil, one has

$$
\frac{q_{s}}{q_{1}}=\frac{\rho_{l, s}}{\rho_{l, 1}}=\frac{C_{w, s}}{C_{w, 1}}=\frac{\theta_{s}}{\theta_{1}}
$$

where the last equality in Eq. (S.18) is derived by assuming that (1) the volumetric moisture content is determined by the total mass of water divided by a constant liquid water density and (2) the amount of water at the soil surface and at the 
middle of the node are put into an identical volume of space, as assumed in Eq. (5) in the main text for a generic tracer.

Substitution of Eq. (S1.8) into Eq. (S1.4) gives

$$
Q=-K\left[\frac{\partial \psi}{\partial \theta}+\left(\frac{\partial \psi}{\partial T} \frac{\partial T}{\partial z}+1\right) \frac{\partial z}{\partial \theta}\right] \theta_{1} \frac{C_{w, s}-C_{w, 1}}{\Delta z / 2}
$$

resulting in an expression for aqueous diffusivity

$$
D_{w, \psi}=-K\left[\frac{\partial \psi}{\partial \theta}+\left(\frac{\partial \psi}{\partial T} \frac{\partial T}{\partial z}+1\right) \frac{\partial z}{\partial \theta}\right]
$$

